\title{
Laparoscopic versus open rectal resection: a 1:2 propensity score-matched analysis of oncological adequateness, short- and long-term outcomes
}

\author{
Giovanni Maria Garbarino ${ }^{1}$ - Giulia Canali ${ }^{1}$. Giulia Tarantino ${ }^{1}$ - Gianluca Costa ${ }^{1}$. Mario Ferri ${ }^{1} \cdot$ Genoveffa Balducci $^{1}$. \\ Emanuela Pilozzi ${ }^{2} \cdot$ Giammauro Berardi $^{1} \cdot$ Paolo Mercantini ${ }^{1}$
}

Accepted: 8 January 2021 / Published online: 22 January 2021

(C) The Author(s) 2021

\begin{abstract}
Background Laparoscopic resections for rectal cancer are routinely performed in high-volume centres. Despite short-term advantages have been demonstrated, the oncological outcomes are still debated. The aim of this study was to compare the oncological adequateness of the surgical specimen and the long-term outcomes between open (ORR) and laparoscopic (LRR) rectal resections.

Methods Patients undergoing laparoscopic or open rectal resections from January 1, 2013, to December 31, 2019, were enrolled. A 1:2 propensity score matching was performed according to age, sex, BMI, ASA score, comorbidities, distance from the anal verge, and clinical $\mathrm{T}$ and $\mathrm{N}$ stage.

Results Ninety-eight ORR were matched to 50 LRR. No differences were observed in terms of operative time (224.9 min. vs. $230.7 ; p=0.567)$ and postoperative morbidity $(18.6 \%$ vs. $20.8 \% ; p=0.744)$. LRR group had a significantly earlier soft oral intake $(p<0.001)$, first bowel movement $(p<0.001)$, and shorter hospital stay $(p<0.001)$. Oncological adequateness was achieved in $85(86.7 \%)$ open and $44(88.0 \%)$ laparoscopic resections $(p=0.772)$. Clearance of the distal $(99.0 \%$ vs. $100 \%$; $p=0.474)$ and radial margins (91.8 vs. $90.0 \%, p=0.709)$, and mesorectal integrity $(94.9 \%$ vs. $98.0 \%, p=0.365)$ were comparable between groups. No differences in local recurrence $(6.1 \%$ vs. $4.0 \%, p=0.589)$, 3-year overall survival $(82.9 \%$ vs. $91.4 \%$, $p=0.276)$, and disease-free survival $(73.1 \%$ vs. $74.3 \%, p=0.817)$ were observed.

Conclusions LRR is associated with good postoperative results, safe oncological adequateness of the surgical specimen, and comparable survivals to open surgery.
\end{abstract}

Keywords Rectal cancer $\cdot$ Laparoscopy $\cdot$ Oncological adequateness $\cdot$ Short-term outcomes $\cdot$ Long-term outcomes

\section{Introduction}

Colorectal cancer (CRC) is the third most common cancer worldwide and the fourth cause of cancer-related deaths. About $35 \%$ of CRC are localized in the rectum [1]. Nowadays, the treatment of CRC is based on a

Paolo Mercantini

paolo.mercantini@uniroma1.it

1 Department of Medical Surgical Science and Translational Medicine, Sapienza University of Rome, Sant'Andrea Hospital, Via di Grottarossa 1035-39, 00189 Rome, Italy

2 Department of Clinical and Molecular Medicine, Pathology Unit, Sapienza University of Rome, Sant'Andrea Hospital, Via di Grottarossa 1035-39, 00189 Rome, Italy multidisciplinary approach which combines surgery, chemotherapy, and radiotherapy. Whenever applicable, curativeintent surgery represents the foundation of CRC as it allows for the complete removal of the tumour and the accurate staging of the disease. Complete excision of the mesorectum containing the lymph nodes and the tumour represents the gold standard to achieve a radical oncological resection and reduce the chance of recurrence within the pelvis $[2,3]$. Surgery is considered oncologically adequate if a clear distal margin and a circumferential radial margin wider than $1 \mathrm{~mm}$ are respected, and a complete mesorectal integrity is obtained [4]. As a matter of fact, patients who benefit from an oncologically adequate resection have an improved local control of the disease and a better long-term survival $[2,4,5]$.

In an effort to minimize the impact of surgery, minimally invasive techniques such as laparoscopy and 
robotics have been implemented in the last 30 years. In this setting, laparoscopic resection for right and left colon cancer has been associated with better short-term outcomes and similar survivals as compared to open [6-12]. Notwithstanding, laparoscopic resection for rectal cancer (LRR) has not been validated yet by international guidelines, unless it is being performed in high-volume centres by experienced surgeons $[1,3]$. Indeed, the rate of conversions reported in the literature is still high, as well as the involvement of the circumferential resection margin [13-15]. The complexity of the pelvic anatomy, the need for a complete excision of the mesorectal fascia, and the clearness of the circumferential margin make minimally invasive rectal surgery much more difficult than open, especially considering lower tumours in a narrow pelvis. Despite this, supporters of the laparoscopic approach speculate that oncological adequateness could still be maintained with safe recurrence rates and survivals, with the benefit of improved short-term outcomes [16, 17].

Due to the lack of consensus, the aim of this study was to compare laparoscopic and open resections for rectal cancer in terms of the oncological adequateness of the surgical specimen and to evaluate the perioperative and oncological outcomes.

\section{Methods}

All consecutive patients undergoing surgery for rectal cancer at the General Surgery Department of Sant'Andrea University Hospital from January 1, 2013, to December 31, 2019, were included in this study. Data were retrospectively reviewed from a prospectively maintained database including demographics, tumour characteristics, operative details, tumour pathology, and shortand long-term outcomes. All patients aged 18 or older, with a histological diagnosis of adenocarcinoma of the rectum within $15 \mathrm{~cm}$ from the anal verge who underwent a rectal resection, were included in the study. Early rectal cancers treated by endoscopic resection, transanal endoscopic microsurgery (TEM), or transanal minimally invasive surgery (TAMIS); advanced cancers invading surrounding structures, metachronous tumours, metastatic cancers requiring major hepatectomies or more than two metastasectomies; patients with a single hepatic metastasis larger than $4 \mathrm{~cm}$ as well as patients with other abdominal malignancies were excluded. Patients were then divided into two groups according to the surgical approach: laparoscopic (LRR group) and open (ORR group) (Fig. 1).

No formal protocol for the allocation of patients to either group was established. The exclusion criteria for the LRR group were medical contraindication to the pneumoperitoneum, hostile abdomen preoperative predictive score $\geq 3,[18]$ previous surgery for endometriosis, previous gastric bypass for obesity, and patient's choice.

The primary endpoint of our study was the oncological adequateness of the resected specimen. As secondary endpoints, perioperative morbidity and mortality and long-term oncological outcomes were evaluated and compared between groups.

All patients were discussed in a multidisciplinary environment involving surgeons, medical oncologists, radiologists, and pathologists. A formal Institutional Review Board approval was not required because of the non-interventional retrospective design; however, a signed consent for treatment and for the analysis of data for scientific purposes was obtained from all patients before surgical intervention.

\section{Definitions and study criteria}

Preoperative clinical staging was assessed by pelvic magnetic resonance imaging (MRI) or endorectal ultrasound in all patients and a total body CT scan was performed in order to detect distant metastases.

Cancers were defined as high $(10-15 \mathrm{~cm})$, middle $(5-$ $10 \mathrm{~cm}$ ), or low $(0-5 \mathrm{~cm})$ rectal tumours according to the distance from the anal verge. Surgical specimens were examined by a single pathologist (E.P). These were received fresh and opened on the anterior wall from the proximal margin up to $4 \mathrm{~cm}$ above the peritoneal reflection for fixation. Before sampling, the integrity of the mesorectum was assessed and the circumferential margin below the peritoneum was inked (Fig. 2). The specimen was then sliced at $1-\mathrm{cm}$ intervals starting from the distal margin. Tumour distance from the circumferential margin was recorded, both macroscopic and microscopic. Lymph nodes close to the circumferential margins were sampled separately. The oncological adequateness of the surgical resection was defined by the contemporary achievement of the following three parameters: (1) a distal resection margin $\geq 1 \mathrm{~mm}$, (2) a circumferential radial margin $(\mathrm{CRM}) \geq 1 \mathrm{~mm}$, and (3) a complete mesorectal fascia integrity. Large defects $(>5 \mathrm{~mm}$ ) of mesorectal fascia were classified as incomplete mesorectal excision. Staging was reported according to the 8th TNM edition [19].

Morbidity and mortality were defined as postoperative complications and death within 30 days from surgery respectively. Morbidity was graded according to the Clavien-Dindo classification and complications graded $>$ II were defined as major [20].

Overall survival (OS) was defined as the time between surgery and death for any cause or last follow-up. Diseasefree survival (DFS) was defined as the time from the operation to tumour recurrence either local or distant.

Neoadjuvant long- or short-course chemo-radiotherapy and adjuvant chemotherapy were administered according to 
Fig. 1 Flow chart of clinical study design (PSM, propensity score matching)

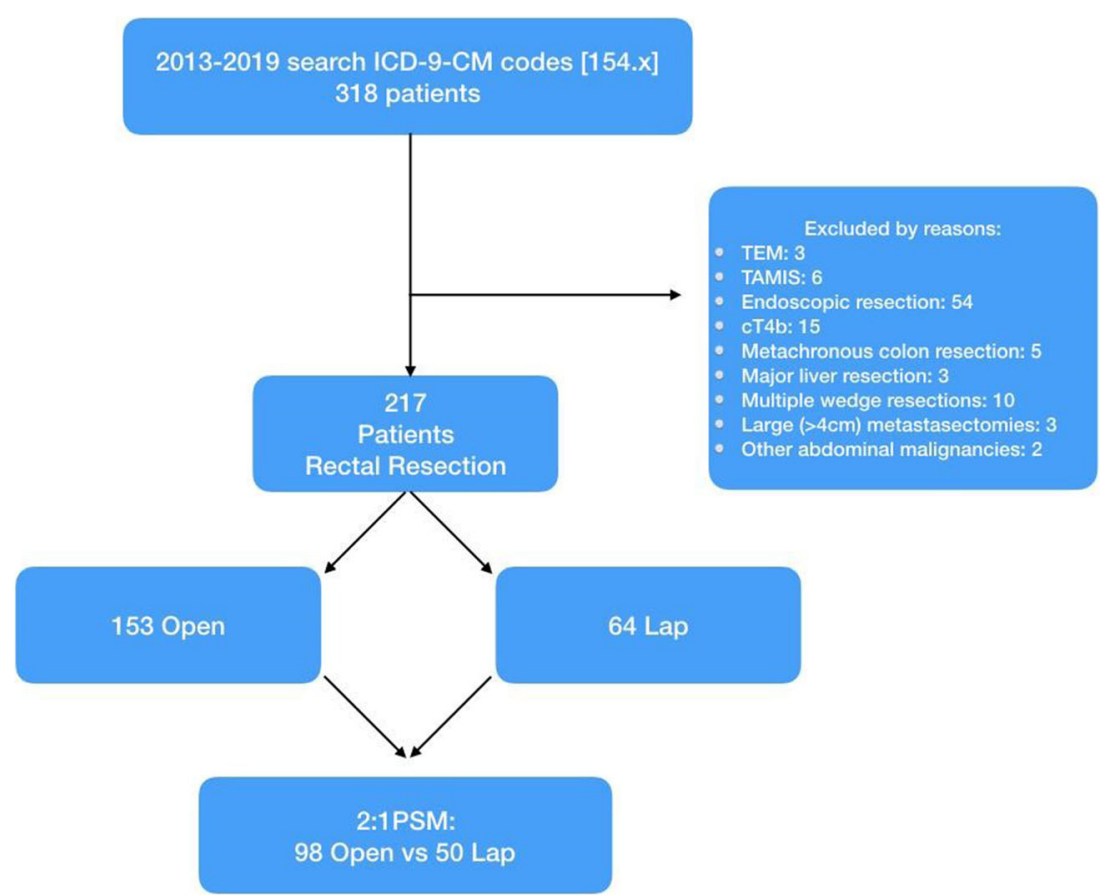

the guidelines of the Italian Society of Medical Oncology (AIOM).

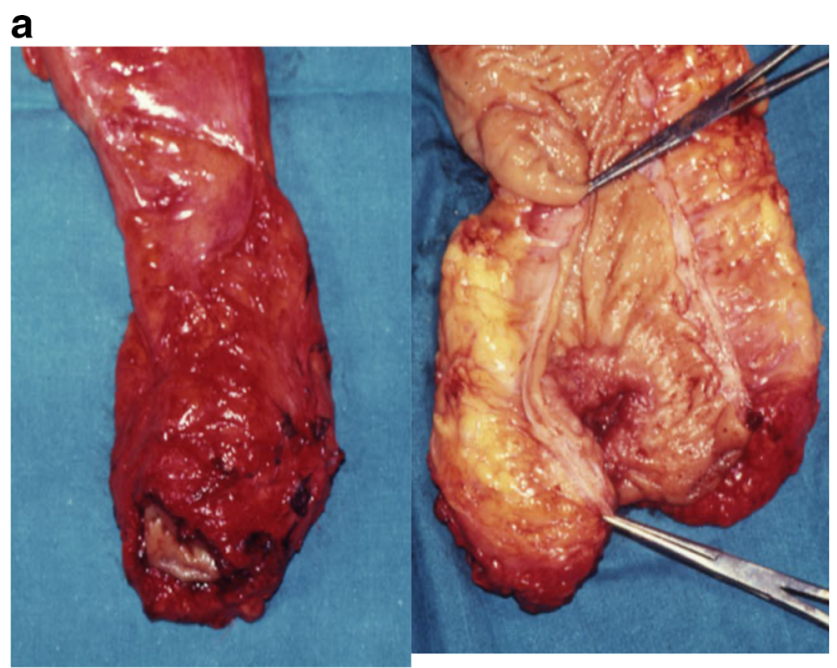

b

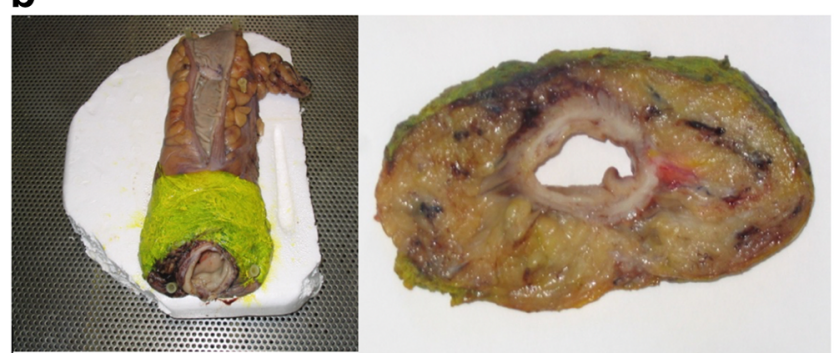

Fig. 2 (A) Complete integrity of the mesorectal fascia; (B) the inked specimen of a total mesorectal excision (TME) and the cross-sectional slice for the evaluation of circumferential resection margin (CRM)

\section{Surgical technique}

Open procedures were performed through a midline laparotomy while a 3 to 4 trocar technique with Pfannesteil incision for the specimen extraction was used in the laparoscopic group. A central ligation of the inferior mesenteric vessels was carried out in both open and laparoscopic cases and the splenic colonic flexure was always taken down. The lateral-to-medial technique was usually preferred in open procedures, whereas medial-to-lateral was more often chosen in laparoscopic surgeries. All the procedures were performed respecting the principles of the total mesorectal excision (TME) [2]. Partial mesorectal excision (PME) was carried out for high rectal tumours whenever a lower margin $\geq 5 \mathrm{~cm}$ was anticipated, avoiding coning resection lines. Diverting or terminal ostomies were reserved for patients with extraperitoneal rectal cancers who underwent neoadjuvant treatment. An end-toend colorectal Knight-Griffen anastomosis was performed both in ORR and LRR cases when intestinal restoration was decided. All surgical procedures both open and laparoscopic were performed by four senior and experienced surgeons.

\section{Statistical analysis}

Continuous data were expressed as the mean ( \pm standard deviation) or median and interquartile range depending on their distribution that was assessed through the Shapiro-Wilk test. An unpaired Student $t$ test was used to compare differences in continuous parametric variables and the Mann-Whitney $U$ test for continuous non-parametric variables. Numbers and percentages were used for reporting categorical variables and 
the $\chi^{2}$ test was used for comparisons. A propensity score matching was applied to eliminate selection bias between groups and reported according to the recommendations of Lonjon et al. [21] Variables influencing decision regarding surgical approach and variables with potential influence on outcomes were assigned propensity scores using a multivariable logistic regression model. The final model included the following variables: age, sex, BMI, ASA score, comorbidity, distance from the anal verge, and clinical $\mathrm{T}$ and $\mathrm{N}$ stage. Setting a calliper width of 0.3 , cases were matched to controls without replacement to the closest matched propensity score with a 1:2 ratio. Laparoscopic procedures converted to open or completion of any part of the pelvic dissection through the site of the specimen extraction were examined according to a perprotocol analysis and included in the ORR group.

Survival analyses were conducted using the Kaplan-Meier method with log-rank test comparisons. Significance was defined as a $p$ value less than 0.05 . Statistical analysis was performed using the SPSS software 25.0 (SPSS, Inc., Chicago, IL).

\section{Results}

Between January 2013 and December 2019, a total of 318 patients underwent a rectal resection for a histologically proven adenocarcinoma at our institution. Three patients who underwent TEM, 6 TAMIS, 54 endoscopic resections, 15 resections of surrounding structures (cT4b), 5 metachronous colonic resections, 3 major liver resections, 10 multiple hepatic wedge resections, 3 metastasectomies larger than $4 \mathrm{~cm}$, and 2 concomitant resections of other abdominal malignancies were excluded from the study. Finally, 217 patients fulfilled the study criteria and were therefore included. One hundred fifty-three (70.5\%) patients underwent an open rectal resection while $64(29.5 \%)$ were operated on by laparoscopy.

\section{Baseline variables before matching}

No significant differences were observed between groups in terms of age, sex, ASA score, BMI, and comorbidities (Table 1). Overall, most of the tumours were located lower than $10 \mathrm{~cm}$ from the anal verge with no differences between ORR and LRR groups (62.7\% vs. $60.9 \% ; p=0.641)$. Larger tumours were operated in the open group compared to laparoscopy $(3.7 \pm 1.9 \mathrm{~cm}$ vs. $3.1 \pm 1.4 \mathrm{~cm} ; p=0.03)$ while preoperative clinical staging was comparable both in terms of $\mathrm{T}$ and $\mathrm{N}$ stage (Table 1). Sixty-two (40.5\%) and $30(46.9 \%)$ patients received neoadjuvant therapy in ORR and LRR groups respectively $(p=0.36)$. Finally, more patients in the ORR group underwent concomitant surgical procedures $(29.4 \%$ vs. $7.8 \%$, $p=0.001$ ) (Table 2). Conversion during LLR occurred in 18 (28.1\%) patients: six were converted early during the operation because of adhesive syndrome, difficulty in the isolation of the inferior mesenteric vessels, or splenic injury during left colonic flexure mobilization with uncontrolled bleeding, and twelve were converted after vessel's ligation and colon mobilization to pursue mesorectal dissection through a 10-12-cm Pfannenstiel incision.

\section{Baseline variables and short-term outcomes after matching}

After propensity score matching, 98 ORR were compared to 50 LRR. Anterior rectal resection was the most common type of operation performed in both groups $(84.7 \%$ in ORR vs. $88 \%$ in LRR; $p=0.71$ ) and a diverting ostomy was chosen in $57(58.1 \%)$ open and $23(46 \%)$ laparoscopic patients $(p=$ $0.34)$. Postoperative outcomes are depicted in Table 2. Time to first flatus was significantly shorter in laparoscopy $(2.5 \pm$ 0.9 in ORR vs. $1.8 \pm 0.9$ in LRR, $p<0.001$ ), as well as time to first bowel movement $(3.5 \pm 1.5$ vs. $2.8 \pm 1.3, p=0.01)$ and time to soft oral intake ( $3.0 \pm 1.3$ vs. $2.0 \pm 1.0$ in, $p<0.001)$. Morbidity was similar between groups $(18.6 \%$ vs. $14.0 \%, p=$ 0.50 ) with most of the complications being minor according to the Clavien-Dindo classification ( $14.4 \%$ in ORR vs. $8.3 \%$ in LRR $p=0.40$ ). Finally, the length of hospital stay was significantly shorter in the laparoscopic group $(11.0 \pm 8.6$ vs. $8.8 \pm$ 7.3 days; $p<0.001$ ).

\section{Pathological and long-term oncological outcomes (Table 3, Fig. 3)}

Pathological examination of the resected specimen showed comparable sizes of the tumour ( 3.6 vs. $3.2 \mathrm{~cm} ; p=0.29$ ), TNM stage $(p=0.96)$, number of retrieved lymph nodes (21.9 vs. $22.3, p=0.96$ ) as well as number of positive nodes between groups. Furthermore, R0 resection was achieved in $89.8 \%$ and $90 \%$ of cases in LRR and ORR respectively $(p=0.56)$. Oncological adequateness of the resected specimen was confirmed in $86.7 \%$ ORR and in $88.0 \%$ LRR with no significant differences $(p=0.772)$. In detail, the distal margin was clear for almost every resection both in open and laparoscopy (99\% vs. $100 \%, p=$ 0.47 ), whereas the circumferential margin was negative in $91.8 \%$ ORR and $90 \%$ LRR $(p=0.70)$. Finally, complete mesorectal integrity was achieved in $94.9 \%$ ORR and in 98\% LRR $(p=0.365)$.

After a median follow-up of 37 (15-61) months, the 3-year actuarial OS rate was $82.9 \%$ for open and $91.4 \%$ for laparoscopy ( $p=0.27$; Fig. 3 ). Six patients $(6.1 \%)$ developed local recurrence following ORR and 2 patients $(4.0 \%)$ after LRR $(p=0.589)$. The 3 -year actuarial DFS rate was $73.1 \%$ in ORR and $74.3 \%$ in LRR ( $p=0.82$; Fig. 3 ). 
Table 1 Patients' characteristics before and after propensity score matching analysis

\begin{tabular}{|c|c|c|c|c|c|c|}
\hline & \multicolumn{3}{|c|}{ Before propensity score matching } & \multicolumn{3}{|c|}{ After propensity score matching } \\
\hline & $\begin{array}{l}\text { ORR } \\
n=153\end{array}$ & $\begin{array}{l}\text { LRR } \\
n=64\end{array}$ & $p$ & $\begin{array}{l}\text { ORR } \\
n=98\end{array}$ & $\begin{array}{l}\mathrm{LRR} \\
n=50\end{array}$ & $p$ \\
\hline $\begin{array}{l}\text { AGE (years, mean, } \pm \\
\text { SD) }\end{array}$ & $66.9( \pm 11.2)$ & $66.5( \pm 12.7)$ & 0.843 & $67.4( \pm 10.9)$ & $66.3( \pm 13.4)$ & 0.664 \\
\hline $\operatorname{SEX}(n, \%)$ & & & 0.650 & & & 0.880 \\
\hline M & $93(60.8 \%)$ & $41(64.1 \%)$ & & $62(63.3 \%)$ & $31(62.0 \%)$ & \\
\hline $\mathrm{F}$ & $60(39.2 \%)$ & $23(35.9 \%)$ & & $36(36.7 \%)$ & $19(38.0 \%)$ & \\
\hline BMI (mean, \pm SD) & $23.3( \pm 3.7)$ & $24.2( \pm 3.8)$ & 0.266 & $24.2( \pm 3.2)$ & $24.2( \pm 4.1)$ & 0.932 \\
\hline $\operatorname{ASA} \operatorname{SCORE}(n, \%)$ & & & 0.865 & & & 0.983 \\
\hline $1-2$ & $66(49.7 \%)$ & $29(47.5 \%)$ & & $46(49.5 \%)$ & $24(51.0 \%)$ & \\
\hline $3-4$ & $67(50.3 \%)$ & $32(52.5 \%)$ & & $47(50.5 \%)$ & $23(48.9 \%)$ & \\
\hline Comorbidities $(n, \%)$ & $68(51.1 \%)$ & $24(47.1 \%)$ & 0.826 & $47(50.5 \%)$ & $24(51.1 \%)$ & 0.858 \\
\hline $\begin{array}{l}\text { Type of } \\
\text { comorbidities }(n, \\
\%)\end{array}$ & & & 0.696 & & & 0.357 \\
\hline Cardiovascular & $41(30.8 \%)$ & $17(58.6 \%)$ & & $33(35.5 \%)$ & $12(25.5 \%)$ & \\
\hline Respiratory & $18(13.5 \%)$ & $6(20.6 \%)$ & & $9(9.7 \%)$ & $9(19.1 \%)$ & \\
\hline Other & $9(6.8 \%)$ & $4(13.8 \%)$ & & $5(5.4 \%)$ & $2(4.3 \%)$ & \\
\hline $\begin{array}{l}\text { Tumour distance } \\
\text { from the anal } \\
\text { verge }(n, \%)\end{array}$ & & & 0.698 & & & 0.536 \\
\hline $10-15 \mathrm{~cm}$ & $57(37.3 \%)$ & $25(39.1 \%)$ & & $34(34.7 \%)$ & $21(42.0 \%)$ & \\
\hline $5-10 \mathrm{~cm}$ & $59(38.6 \%)$ & $21(31.8 \%)$ & & $36(36.7 \%)$ & $14(28.0 \%)$ & \\
\hline $0-5 \mathrm{~cm}$ & $37(24.2 \%)$ & $18(28.1 \%)$ & & $28(28.6 \%)$ & $15(30.0 \%)$ & \\
\hline Tumour size (cm) & $3.7( \pm 1.9)$ & $3.1( \pm 1.4)$ & 0.034 & $3.6( \pm 1.8)$ & $3.2( \pm 1.4)$ & 0.290 \\
\hline $\begin{array}{l}\text { mean, } \pm \text { SD } \\
\text { Median (IQR } \\
\text { 25-75\%) }\end{array}$ & $3(2.4-4.5)$ & $3(2.0-3.9)$ & & $3(2.2-4.5)$ & $3(2.2-4.0)$ & \\
\hline $\begin{array}{l}\text { Clinical T-stage ( } n \text {, } \\
\%)\end{array}$ & & & 0.372 & & & 0.999 \\
\hline $\mathrm{cT} 1$ & $21(13.7 \%)$ & $10(15.7 \%)$ & & $9(9.2 \%)$ & $5(10.0 \%)$ & \\
\hline $\mathrm{cT} 2$ & $32(20.9 \%)$ & $12(18.8 \%)$ & & $21(21.4 \%)$ & $11(22.0 \%)$ & \\
\hline cT3 & $79(51.6 \%)$ & $37(57.8 \%)$ & & $60(61.2 \%)$ & $30(60.0 \%)$ & \\
\hline cT4 & $21(13.7 \%)$ & $5(7.8 \%)$ & & $8(8.2 \%)$ & $4(8.0 \%)$ & \\
\hline $\begin{array}{l}\text { Clinical N-stage ( } n \text {, } \\
\%)\end{array}$ & & & 0.736 & & & 0.866 \\
\hline cN0 & $79(51.6 \%)$ & $39(60.9 \%)$ & & $52(53.1 \%)$ & $30(60.0 \%)$ & \\
\hline $\mathrm{cN} 1$ & $45(29.4 \%)$ & $15(23.4 \%)$ & & $28(28.6 \%)$ & $12(24.0 \%)$ & \\
\hline $\mathrm{cN} 2$ & $29(18.9 \%)$ & $10(15.6 \%)$ & & $18(18.4 \%)$ & $8(16.0 \%)$ & \\
\hline $\begin{array}{l}\text { Neoadjuvant therapy } \\
\quad(n, \%)\end{array}$ & & & 0.364 & & & 0.937 \\
\hline $\begin{array}{l}\text { Standard RT + } \\
\text { CHT }\end{array}$ & $51(33.3 \%)$ & $25(39.1 \%)$ & & $35(35.7 \%)$ & $17(34.0 \%)$ & \\
\hline Short RT & $11(7.2 \%)$ & $5(7.8 \%)$ & & $9(9.2 \%)$ & $4(8.0 \%)$ & \\
\hline
\end{tabular}

\section{Oncological adequateness of rectal resections for tumours below the peritoneal reflection (Table 4)}

Two subgroups were further analysed according to the location of the tumour below the peritoneal reflection: the middle rectum group (5-10 $\mathrm{cm}$ from the anal verge) and the low rectum group (0-5 $\mathrm{cm}$ from the anal verge). Patients in both groups underwent a total mesorectal excision and the oncological adequateness of the resection of middle versus low rectal tumours was comparable regardless of the surgical technique $(84.0 \%$ in middle vs. $76.7 \%$ in low rectal cancers; $p=0.37$ ).

Comparing ORR and LRR, the oncological adequateness was comparable both in the middle rectum group $(83.3 \%$ vs. $85.7 \%$, $p=0.837)$ and in the low rectum group (75.0\% vs. $80.0 \%, p=$ 
Table 2 Perioperative outcomes before and after propensity score matching

\begin{tabular}{|c|c|c|c|c|c|c|}
\hline & \multicolumn{3}{|c|}{ Before propensity score matching } & \multicolumn{3}{|c|}{ After propensity score matching } \\
\hline & $\begin{array}{l}\text { ORR } \\
n=153\end{array}$ & $\begin{array}{l}\text { LRR } \\
n=64\end{array}$ & $p$ & $\begin{array}{l}\text { ORR } \\
n=98\end{array}$ & $\begin{array}{l}\text { LRR } \\
n=50\end{array}$ & $p$ \\
\hline Operative time (min, mean $\pm \mathrm{SD}$ ) & $220.4 \pm 60.8$ & $226.9 \pm 57.3$ & 0.464 & $224.9 \pm 63.5$ & $230.7 \pm 58.8$ & 0.567 \\
\hline \multicolumn{7}{|l|}{ Type of resection ( $n$ pts, \%) } \\
\hline Anterior resection & $136(88.9 \%)$ & $56(87.5 \%)$ & 0.623 & $83(84.7 \%)$ & $44(88.0 \%)$ & 0.712 \\
\hline Abdominoperineal resection & $14(9.2 \%)$ & $8(12.5 \%)$ & & $14(14.3 \%)$ & $6(12.0 \%)$ & \\
\hline Hartmann & $3(1.9 \%)$ & $0(0.0 \%)$ & & $1(1.0 \%)$ & $0(0.0 \%)$ & \\
\hline Associated procedure ( $n$ pts, $\%$ ) & $45(29.4 \%)$ & $5(7.8 \%)$ & 0.001 & $24(24.5 \%)$ & $5(10.0 \%)$ & 0.144 \\
\hline Cholecistectomy & $4(2.6 \%)$ & $1(1.6 \%)$ & & $3(3.1 \%)$ & $1(2.0 \%)$ & \\
\hline $\begin{array}{l}\text { Hepatic metastasectomy } \\
(1 \text { metastasis })\end{array}$ & $4(2.6 \%)$ & $1(1.6 \%)$ & & $1(1.0 \%)$ & $1(2.0 \%)$ & \\
\hline $\begin{array}{l}\text { Hepatic metastasectomy } \\
(2 \text { metastases) }\end{array}$ & $3(1.9 \%)$ & $0(0.0 \%)$ & & $2(2.0 \%)$ & $0(0.0 \%)$ & \\
\hline Liver biopsy & $4(2.6 \%)$ & $0(0.0 \%)$ & & $1(1.0 \%)$ & $0(0.0 \%)$ & \\
\hline Right or left annessiectomy & $9(5.9 \%)$ & $1(1.6 \%)$ & & $5(5.1 \%)$ & $1(2.0 \%)$ & \\
\hline Uterine myomectomy & $1(0.7 \%)$ & $0(0.0 \%)$ & & $0(0.0 \%)$ & $0(0.0 \%)$ & \\
\hline Splenectomy & $3(1.9 \%)$ & $0(0.0 \%)$ & & $2(2.0 \%)$ & $0(0.0 \%)$ & \\
\hline Appendectomy & $8(5.2 \%)$ & $0(0.0 \%)$ & & $5(5.1 \%)$ & $0(0.0 \%)$ & \\
\hline Meckel's diverticulum resection & $1(0.7 \%)$ & $0(0.0 \%)$ & & $1(1.0 \%)$ & $0(0.0 \%)$ & \\
\hline Colostomy reversal & $2(1.3 \%)$ & $1(1.6 \%)$ & & $1(1.0 \%)$ & $1(2.0 \%)$ & \\
\hline Abdominal hernia repair & $5(3.3 \%)$ & $1(1.6 \%)$ & & $1(1.0 \%)$ & $1(2.0 \%)$ & \\
\hline Ureteral injury repair & $1(0.7 \%)$ & $0(0.0 \%)$ & & $1(1.0 \%)$ & $0(0.0 \%)$ & \\
\hline Ostomy ( $n$ pts, \%) & & & 0.673 & & & 0.342 \\
\hline Ileostomy & $60(39.6 \%)$ & $21(32.8 \%)$ & & $40(40.8 \%)$ & $15(30.0 \%)$ & \\
\hline Colostomy & $24(15.7 \%)$ & $11(17.2 \%)$ & & $17(17.3 \%)$ & $8(16.0 \%)$ & \\
\hline Time to first flatus (days, mean \pm SD) & $2.5 \pm 0.9$ & $1.8 \pm 0.9$ & $<0.001$ & $2.5 \pm 0.9$ & $1.8 \pm 0.9$ & $<0.001$ \\
\hline Time to first bowel movement (days, mean $\pm \mathrm{SD}$ ) & $3.6 \pm 1.6$ & $2.8 \pm 1.3$ & 0.009 & $3.5 \pm 1.5$ & $2.8 \pm 1.3$ & 0.019 \\
\hline Time to soft oral intake (days, mean $\pm \mathrm{SD}$ ) & $3.0 \pm 1.2$ & $2.0 \pm 1.0$ & $<0.001$ & $3.0 \pm 1.3$ & $2.0 \pm 1.0$ & $<0.001$ \\
\hline 30-days morbidity ( $n$ pts, $\%$ ) & $34(23.2 \%)$ & $10(19.6 \%)$ & 0.596 & $17(17.3 \%)$ & $7(14.0 \%)$ & 0.502 \\
\hline Clavien-Dindo classification ( $n$ pts, $\%$ ) & & & 0.017 & & & 0.401 \\
\hline $\mathrm{I}-\mathrm{II}$ & $26(17.0 \%)$ & $4(6.2 \%)$ & & $14(14.3 \%)$ & $4(8.3 \%)$ & \\
\hline III-IV & $8(5.2 \%)$ & $6(9.4 \%)$ & & $3(3.1 \%)$ & $3(6.0 \%)$ & \\
\hline \multicolumn{7}{|l|}{ Type of complication } \\
\hline Anastomotic bleeding & $0(0.0 \%)$ & $1(1.6 \%)$ & & $0(0.0 \%)$ & $1(2.0 \%)$ & \\
\hline Anastomotic leak & $11(7.2 \%)$ & $5(7.8 \%)$ & & $6(6.1 \%)$ & $3(6.0 \%)$ & \\
\hline Intra-abdominal bleeding & $2(1.3 \%)$ & $0(0.0 \%)$ & & $0(0.0 \%)$ & $0(0.0 \%)$ & \\
\hline Canalization delay & $3(1.9 \%)$ & $2(3.1 \%)$ & & $2(2.0 \%)$ & $1(2.0 \%)$ & \\
\hline Neurological & $0(0.0 \%)$ & $0(0.0 \%)$ & & $0(0.0 \%)$ & $0(0.0 \%)$ & \\
\hline Cardiac & $2(1.3 \%)$ & $0(0.0 \%)$ & & $1(1.0 \%)$ & $0(0.0 \%)$ & \\
\hline Pulmonary & $6(3.9 \%)$ & $0(0.0 \%)$ & & $3(3.1 \%)$ & $0(0.0 \%)$ & \\
\hline Urinary & $3(1.9 \%)$ & $0(0.0 \%)$ & & $2(2.0 \%)$ & $0(0.0 \%)$ & \\
\hline Wound infection & $3(1.9 \%)$ & $0(0.0 \%)$ & & $2(2.0 \%)$ & $0(0.0 \%)$ & \\
\hline Other & $4(2.6 \%)$ & $2(3.1 \%)$ & & $1(1.0 \%)$ & $2(4.0 \%)$ & \\
\hline Length of stay (days, mean \pm SD) & $11.5 \pm 8.3$ & $8.6 \pm 7.4$ & $<0.001$ & $11.0 \pm 8.6$ & $8.8 \pm 7.3$ & $<0.001$ \\
\hline 30-day mortality ( $n$ pts, $\%$ ) & $1(0.7 \%)$ & $2(3.8 \%)$ & 0.155 & $1(1.0 \%)$ & $1(2.1 \%)$ & 0.625 \\
\hline
\end{tabular}

0.71). Furthermore, no statistically significant differences were observed between ORR and LRR patients regarding the distal and circumferential clearance and mesorectal integrity for both middle and low rectum groups (Table 4). 
Table 3 Pathological data before and after propensity score matching analysis

\begin{tabular}{|c|c|c|c|c|c|c|}
\hline \multirow[t]{2}{*}{ Oncologic data } & \multicolumn{3}{|c|}{ Before propensity score matching } & \multicolumn{3}{|c|}{ After propensity score matching } \\
\hline & $\begin{array}{l}\text { ORR } \\
n=153\end{array}$ & $\begin{array}{l}\text { LRR } \\
n=64\end{array}$ & $p$ & $\begin{array}{l}\text { ORR } \\
n=98\end{array}$ & $\begin{array}{l}\text { LRR } \\
n=50\end{array}$ & $p$ \\
\hline pTNM stage & & & 0.598 & & & 0.965 \\
\hline y0 & $12(7.9 \%)$ & $3(4.7 \%)$ & & $3(3.1 \%)$ & $24.0 \%)$ & \\
\hline I & $27(17.6 \%)$ & $14(21.9 \%)$ & & $19(19.4 .5 \%)$ & $1122.0 \%)$ & \\
\hline II & $38(24.8 \%)$ & $17(26.5 \%)$ & & $28(28.6 \%)$ & $13(26 \%)$ & \\
\hline III & $65(42.5 \%)$ & $28(43.8 \%)$ & & $44(44.9 \%)$ & $22(44.0 \%)$ & \\
\hline IV & $11(7.2)$ & $2(3.1 \%)$ & & $4(4.1 \%)$ & $2(4.0 \%)$ & \\
\hline Retrieved nodes (mean $\pm \mathrm{SD}$ ) & $21.5 \pm 13.0$ & $20.4 \pm 13.6$ & 0.435 & $21.9 \pm 12.8$ & $22.3 \pm 13.7$ & 0.960 \\
\hline Positive nodes (mean $\pm \mathrm{SD})$ & $2.1 \pm 4.0$ & $1.3 \pm 2.5$ & 0.176 & $2.3 \pm 4.6$ & $1.46 \pm 2.7$ & 0.387 \\
\hline Resection margin $(n, \%)$ & & & 0.246 & & & 0.563 \\
\hline $\mathrm{R} 0$ & $139(90.8 \%)$ & $57(89.1)$ & & $88(89.8 \%)$ & $45(90.0 \%)$ & \\
\hline $\mathrm{R} 1$ & $10(6.5 \%)$ & $7(10.9 \%)$ & & $8(8.2 \%)$ & $5(10.0 \%)$ & \\
\hline $\mathrm{R} 2$ & $4(2.6 \%)$ & $0(0.0 \%)$ & & $2(2.0 \%)$ & $0(0.0 \%)$ & \\
\hline Oncological adequateness $(n, \%)$ & & & 0.742 & & & 0.772 \\
\hline Yes & $134(87.6 \%)$ & $55(85.9 \%)$ & & $85(86.7 \%)$ & $44(88.0 \%)$ & \\
\hline No & $19(12.4 \%)$ & $9(14.4 \%)$ & & $13(13.3 \&)$ & $6(12.0 \%)$ & \\
\hline Mesorectal fascia integrity $(n, \%)$ & & & 0.625 & & & 0.365 \\
\hline Complete & $146(95.4 \%)$ & $62(96.9 \%)$ & & $93(94.9 \%)$ & $49(98.0 \%)$ & \\
\hline Incomplete & $7(4.6 \%)$ & $2(3.1 \%)$ & & $5(5.1 \%)$ & $1(2.0 \%)$ & \\
\hline Distal margin $(n, \%)$ & & & 0.517 & & & 0.474 \\
\hline$\geq 1 \mathrm{~mm}$ & $152(99.3 \%)$ & $64(100 \%)$ & & $97(99.0 \%)$ & $50(100 \%)$ & \\
\hline$<1$ & $1(0.7 \%)$ & $0(0.0 \%)$ & & $1(1.0 \%)$ & $0(0.0 \%)$ & \\
\hline Circumferential margin $(n, \%)$ & & & 0.462 & & & 0.709 \\
\hline$\geq 1 \mathrm{~mm}$ & $141(92.2 \%)$ & $57(89.1 \%)$ & & $90(91.8 \%)$ & $45(90.0 \%)$ & \\
\hline$<1 \mathrm{~mm}$ & $12(7.8 \%)$ & $7(10.9 \%)$ & & $8(8.2 \%)$ & $5(10.0 \%)$ & \\
\hline
\end{tabular}

\section{Discussion}

Laparoscopic resection of rectal cancer is still controversial. Despite the short-term benefits that have been widely accepted

a

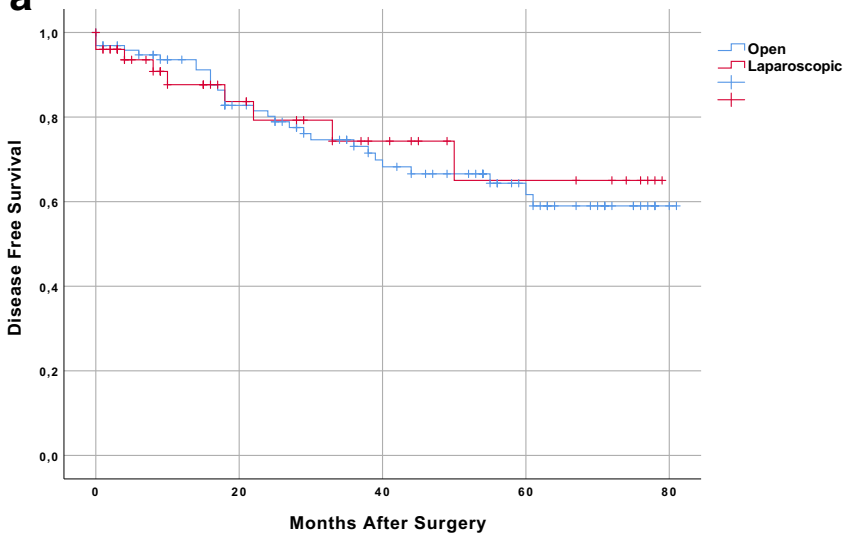

$[14,17,22]$, the oncological adequateness and the long-term survivals following LRR are still debated. Previous randomized clinical trials showed no differences between the open and laparoscopic approaches in terms of oncological

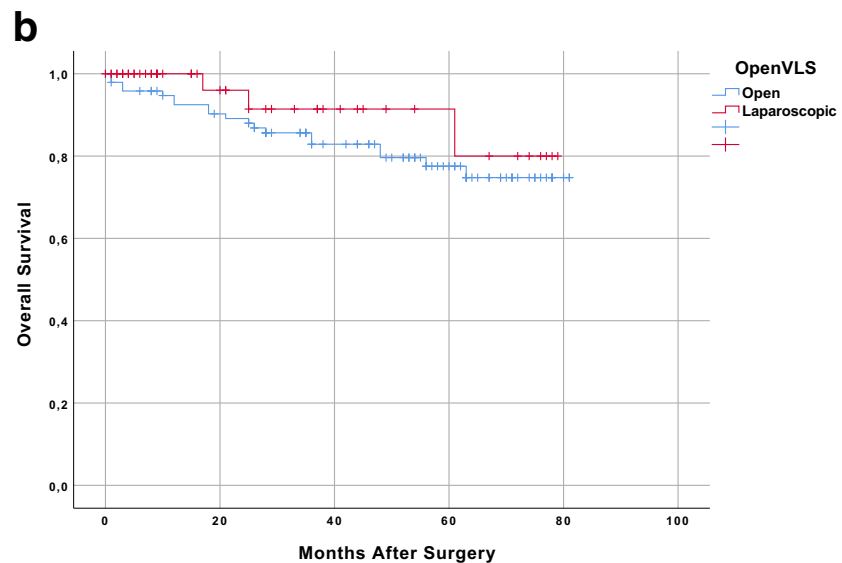

Fig. 3 (A) Patient's overall survival curves according to surgical approach; (B) patient's disease-free survival curves according to surgical approach 
Table 4 Subgroups analysis of the oncological adequateness of middle and low rectal cancers resections after propensity score matching analysis

\begin{tabular}{|c|c|c|c|c|c|c|}
\hline & \multicolumn{3}{|c|}{ Middle rectum } & \multicolumn{3}{|l|}{ Low rectum } \\
\hline & \multicolumn{3}{|l|}{$(N=50)$} & \multicolumn{3}{|l|}{$(N=43)$} \\
\hline & $\begin{array}{l}\text { ORR } \\
(N=36)\end{array}$ & $\begin{array}{l}\text { LRR } \\
(N=14)\end{array}$ & $p$ value & $\begin{array}{l}\text { ORR } \\
(N=28)\end{array}$ & $\begin{array}{l}\text { LRR } \\
(N=15)\end{array}$ & $p$ value \\
\hline Mesorectal fascia integrity, $n(\%)$ & $34(94.4 \%)$ & $14(100 \%)$ & 0.368 & $25(89.3 \%)$ & $14(93.3 \%)$ & 0.663 \\
\hline Distal clearance, $n(\%)$ & $35(97.2 \%)$ & $14(100 \%)$ & 0.529 & $28(100 \%)$ & $15(100 \%)$ & 1.000 \\
\hline Radial clearance, $n(\%)$ & $33(91.7 \%)$ & $12(85.7 \%)$ & 0.529 & $23(82.1 \%)$ & $13(86.7 \%)$ & 0.702 \\
\hline Oncological adequateness, $n(\%)$ & $30(83.3 \%)$ & $12(85.7 \%)$ & 0.837 & $21(75.0 \%)$ & $12(80.0 \%)$ & 0.711 \\
\hline Locoregional recurrence, $n(\%)$ & $1(2.8 \%)$ & $0(0.0 \%)$ & 0.529 & $4(14.3 \%)$ & $1(6.7 \%)$ & 0.458 \\
\hline
\end{tabular}

adequateness of the surgical specimen; notwithstanding, recently, the ACOSOG Z6051 and the ALaCaRT trial failed to demonstrate the non-inferiority of the laparoscopic technique [13-17]. Indeed, despite long-term results, it showed similar OS, DFS, and locoregional recurrences, higher rates of CRM involvement were shown in laparoscopy [14, 15, 23]. Nevertheless, surgeries not respecting the three pathologic criteria (clearance of the distal and radial margins and integrity of the mesorectal fascia) and therefore failing to achieve oncological adequacy were associated with shorter DFS and increased locoregional recurrence, being the involvement of CRM, the most important parameter influencing survival $[24,25]$. In the ongoing debate, the current manuscript demonstrates the oncological adequateness of laparoscopic rectal resection using a propensity score matching analysis that, besides randomisation, represents the best available method to reduce selection bias when analysing the outcomes related to surgical techniques, therefore obtaining high-quality and reliable evidence. No differences in terms of adequacy of the oncological specimen were found between ORR and LRR respecting the oncological criteria in most of the resections and for both approaches. These pathological results eventually translated into comparable OS, DFS, and locoregional recurrence, strengthening the importance of adequate rectal resections in the oncological long-term prognosis of patients. As an additional finding, in our study, we have shown that considering only extraperitoneal tumours, ORR and LRR had a comparable clearance of distal and circumferential margins and mesorectal integrity, defining the resected specimen as oncologically adequate in both approaches. As a matter of fact, during laparoscopic resections for middle and low rectal cancers, advanced technical skills are required to ensure appropriate quality of the operation, especially in the setting of a narrow pelvis, neoadjuvant treatment, large tumours, and/or a high BMI. Indeed, in the above-mentioned scenarios, the adequacy of the resected specimen is much more difficult to obtain, eventually requiring technical proficiency to avoid coning effect and ensure the safety of the procedure both postoperatively and oncologically [14]. Notwithstanding, according to our results, LLRs could be considered oncologically adequate even for middle and low rectal tumours. Robotic surgery and transanal total mesorectal excision (TaTME) were recently developed to overcome some technical issues of standard laparoscopy during technically demanding resections, especially in middle and low rectal resections. The robotic approach seems to ensure wider distal margins as compared to LRR whereas TaTME is helpful to achieve a wider CRM and a complete grading of mesorectal quality [26-32]. Despite this, the ROLARR trial has recently demonstrated that robotic-assisted surgery does not confer an advantage over pure laparoscopy [28] whereas TaTME was recently halted in Norway as part of a national policy because of the early and high recurrence rates [33]. Therefore, emphasis and further evidence should still be encouraged in the setting of laparoscopy as it is surely the most used and widely available technique nowadays.

Minimally invasive resections have been shown as beneficial in terms of postoperative outcomes in different surgical settings [34-36]. Even in colorectal surgery, short-term outcomes are significantly improved when compared to open surgery [14, 17, 22]. First flatus, bowel canalization, and soft oral intake happened significantly earlier in LRR in our study and hospital stay was significantly shorter [14, 17, 22]. No significant differences in terms of postoperative morbidity were observed between groups; nonetheless, ORRs were associated with higher rates of minor complications especially considering wound infections, pneumonia, and urinary tract infections. Larger incisions and slower recovery after surgery are surely contributing and probably affecting both patient's postoperative period and hospital discharge. In this setting, the role of enhanced recovery after surgery further improves the short-term results of minimally invasive techniques and should therefore be encouraged [37-39].

While laparoscopy currently represents the standard approach for the treatment of right and left colon cancers, resections of the rectum are technically demanding and require an 
appropriate learning curve, selection of patients, and referral centres. Comparing the two populations of our study before propensity score matching, patients undergoing laparoscopic or open rectal resections were overall comparable in terms of demographics and clinical presentation. Certainly, the LRR group counts fewer patients as a result of the learning curve of a relatively novel technique, but the absence of differences in the unmatched cohorts suggests that selection bias in our experience was not significant even before correction. In our centre, indeed, we tend to approach all rectal tumours by laparoscopy, excluding only cases in which tumour invades surrounding structures (T4b) or in which an associated major procedure is preoperatively planned (i.e. metastatic disease requiring contemporary liver resections). Notwithstanding, we still matched the groups using propensity score analysis to correct for undetectable selection bias eventually improving the statistical power of the study.

This study has some limitations mainly being the retrospective fashion and the relatively small sample size. The study was limited to the most recent 5-year period to avoid bias induced by the learning curve effect. Indeed, in 2013, the 4 surgeons were already all experienced in the laparoscopic colon and rectal resections reducing the bias of surgical technique. Furthermore, they independently performed both open and laparoscopic procedures.

\section{Conclusions}

Laparoscopic surgery for rectal cancer is associated with good postoperative results. Compared to the open approach, oncological adequateness of the resected specimen is respected, even in the setting of middle and low rectal tumours eventually ensuring long-term survivals. Surgeons in high-volume centres should be encouraged to perform more laparoscopic resections in order to provide improved postoperative recovery maintaining safe long-term survival.

Funding Open Access funding provided by Università degli Studi di Roma La Sapienza.

\section{Compliance with ethical standards}

Conflict of interest The authors declare that they have no conflict of interest.

Open Access This article is licensed under a Creative Commons Attribution 4.0 International License, which permits use, sharing, adaptation, distribution and reproduction in any medium or format, as long as you give appropriate credit to the original author(s) and the source, provide a link to the Creative Commons licence, and indicate if changes were made. The images or other third party material in this article are included in the article's Creative Commons licence, unless indicated otherwise in a credit line to the material. If material is not included in the article's Creative Commons licence and your intended use is not permitted by statutory regulation or exceeds the permitted use, you will need to obtain permission directly from the copyright holder. To view a copy of this licence, visit http://creativecommons.org/licenses/by/4.0/.

\section{References}

1. Glynne-Jones R, Wyrwicz L, Tiret E et al (2017) Rectal cancer: ESMO Clinical Practice Guidelines for diagnosis, treatment and follow-up. Ann Oncol 28:iv22-iv40

2. Heald RJ, Husband EM, Ryall RDH (1982) The mesorectum in rectal cancer surgery - the clue to pelvic recurrence? Br J Surg 69:613-616

3. Benson AB, Venook AP, Al-Hawary MM et al (2018) Rectal cancer, version 2.2018 clinical practice guidelines in oncology. JNCCN J Natl Compr Cancer Netw 16:874-901

4. Monson JRT, Weiser MR, Buie WD, Chang GJ, Rafferty JF, Buie WD, Rafferty J, Guillem J, Boushey R, Chang G, Feingold D, Fleshner P, Genua J, Hammond K, Harb W, Hendren S, Herzig D, Kaiser A, Larson D, Lee S, McCormick J, Melton-Meaux G, Mills S, Monson J, Moore H III, Perry WB, Phang PT, Rivadeneira D, Ross H, Steele S, Strong S, Ternent C, Varma M, Weiser M, Wilkins K (2013) Practice parameters for the management of rectal cancer (revised). Dis Colon Rectum 56:535-550

5. MacFarlane JK, Ryall RDH, Heald RJ (1993) Mesorectal excision for rectal cancer. Lancet 341:457-460

6. Schwenk W, Haase O, Neudecker JJ, Müller JM, Cochrane Colorectal Cancer Group (2005) Short term benefits for laparoscopic colorectal resection. Cochrane Database Syst Rev. https:// doi.org/10.1002/14651858.cd003145.pub2

7. Noel JK, Fahrbach K, Estok R, Cella C, Frame D, Linz H, Cima RR, Dozois EJ, Senagore AJ (2007) Minimally invasive colorectal resection outcomes: short-term comparison with open procedures. J Am Coll Surg 204:291-307

8. Zhao D, Li Y, Wang S, Huang Z (2016) Laparoscopic versus open surgery for rectal cancer: a meta-analysis of 3-year follow-up outcomes. Int J Color Dis 31:805-811

9. Nelson H, Sargent DJ, Wieand HS et al (2004) A comparison of laparoscopically assisted and open colectomy for colon cancer. $\mathrm{N}$ Engl J Med 350:2050-2059

10. Jacob BP, Salky B (2005) Laparoscopic colectomy for colon adenocarcinoma: an 11-year retrospective review with 5-year survival rates. Surg Endosc Other Interv Tech 19:643-649

11. Nakamura T, Mitomi H, Ohtani Y et al (2006) Comparison of longterm outcome of laparoscopic and conventional surgery for advanced colon and rectosigmoid cancer. Hepatogastroenterology 53:351-353

12. Colon Cancer Laparoscopic or Open Resection Study Group, Buunen M, Veldkamp R et al (2009) Survival after laparoscopic surgery versus open surgery for colon cancer: long-term outcome of a randomised clinical trial. Lancet Oncol 10:44-52

13. Green BL, Marshall HC, Collinson F, Quirke P, Guillou P, Jayne DG, Brown JM (2013) Long-term follow-up of the Medical Research Council CLASICC trial of conventional versus laparoscopically assisted resection in colorectal cancer. Br J Surg 100:75-82

14. Stevenson ARL, Solomon MJ, Lumley JW, Hewett P, Clouston AD, Gebski VJ, Davies L, Wilson K, Hague W, Simes J (2015) Effect of laparoscopic-assisted resection vs open resection on pathological outcomes in rectal cancer: the ALaCaRT randomized clinical trial. JAMA - J Am Med Assoc 314:1356-1363

15. Fleshman J, Branda M, Sargent DJ et al (2016) Effect of laparoscopic-assisted resection vs open resection of stage II or III 
rectal cancer on pathologic outcomes. JAMA - J Am Med Assoc 314:1346-1355

16. Jeong SY, Park JW, Nam BH, Kim S, Kang SB, Lim SB, Choi HS, Kim DW, Chang HJ, Kim DY, Jung KH, Kim TY, Kang GH, Chie EK, Kim SY, Sohn DK, Kim DH, Kim JS, Lee HS, Kim JH, Oh JH (2014) Open versus laparoscopic surgery for mid-rectal or lowrectal cancer after neoadjuvant chemoradiotherapy (COREAN trial): survival outcomes of an open-label, non-inferiority, randomised controlled trial. Lancet Oncol 15:767-774

17. Bonjer HJ, Deijen CL, Abis GA, Cuesta MA, van der Pas MHGM, de Lange-de Klerk ESM, Lacy AM, Bemelman WA, Andersson J, Angenete E, Rosenberg J, Fuerst A, Haglind E (2015) A randomized trial of laparoscopic versus open surgery for rectal cancer. $\mathrm{N}$ Engl J Med 372:1324-1332

18. Goldfarb MA, Protyniak B, Schultheis M (2014) Hostile abdomen index risk stratification and laparoscopic complications. JSLS, J Soc Laparoendosc Surg 18:14-19

19. Amin M, Edge S, Al E (2017) AJCC Cancer Staging Manual, 8th edn, New York

20. Clavien PA, Barkun J, De Oliveira ML et al (2009) The ClavienDindo classification of surgical complications: five-year experience. Ann Surg 250:187-196

21. Lonjon G, Porcher R, Ergina P, Fouet M, Boutron I (2017) Potential pitfalls of reporting and bias in observational studies with propensity score analysis assessing a surgical procedure: a methodological systematic review. Ann Surg 265:901-909

22. Lujan J, Valero G, Hernandez Q, Sanchez A, Frutos MD, Parrilla P (2009) Randomized clinical trial comparing laparoscopic and open surgery in patients with rectal cancer. Br J Surg 96:982-989

23. Jayne DG, Guillou PJ, Thorpe H, Quirke P, Copeland J, Smith AM, Heath RM, Brown JM, UK MRC CLASICC Trial Group (2007) Randomized trial of laparoscopic-assisted resection of colorectal carcinoma: 3-year results of the UK MRC CLASICC trial group. J Clin Oncol 25:3061-3068

24. Stevenson ARL, Solomon MJ, Brown CSB, Lumley JW, Hewett P, Clouston AD, Gebski VJ, Wilson K, Hague W, Simes J, Australasian Gastro-Intestinal Trials Group (AGITG) ALaCaRT investigators (2019) Disease-free survival and local recurrence after laparoscopic-assisted resection or open resection for rectal cancer: the Australasian laparoscopic cancer of the rectum randomized clinical trial. Ann Surg 269:596-602

25. Fleshman J, Branda ME, Sargent DJ, Boller AM, George VV, Abbas MA, Peters WR Jr, Maun DC, Chang GJ, Herline A, Fichera A, Mutch MG, Wexner SD, Whiteford MH, Marks J, Birnbaum E, Margolin DA, Larson DW, Marcello PW, Posner MC, Read TE, Monson JRT, Wren SM, Pisters PWT, Nelson H (2019) Disease-free survival and local recurrence for laparoscopic resection compared with open resection of stage II to III rectal cancer: follow-up results of the ACOSOG Z6051 randomized controlled trial. Ann Surg 269:589-595

26. Liao G, Zhao Z, Deng H et al Comparison of pathological outcomes between robotic rectal cancer surgery and laparoscopic rectal cancer surgery: A meta-analysis based on seven randomized controlled trials. Int J Med Robot Comput Assist Surg 15. https://doi.org/10. 1002/rcs.2027

27. Han C, Yan P, Jing W et al Clinical, pathological, and oncologic outcomes of robotic-assisted versus laparoscopic proctectomy for rectal cancer: a meta-analysis of randomized controlled studies. Asian J Surg. https://doi.org/10.1016/j.asjsur.2019.11.003

28. Jayne D, Pigazzi A, Marshall H, Croft J, Corrigan N, Copeland J, Quirke P, West N, Edlin R, Hulme C, Brown J (2019) Roboticassisted surgery compared with laparoscopic resection surgery for rectal cancer: the ROLARR RCT. Effic Mech Eval 6:1-140

29. Roodbeen SX, De Lacy FB, Van Dieren S et al (2019) Predictive factors and risk model for positive circumferential resection margin rate after transanal total mesorectal excision in 2653 patients with rectal cancer. Ann Surg. https://doi.org/10.1097/SLA. 0000000000003516

30. van Oostendorp SE, Belgers HJ, Bootsma BT, Hol JC, Belt EJTH, Bleeker W, den Boer FC, Demirkiran A, Dunker MS, Fabry HFJ, Graaf EJR, Knol JJ, Oosterling SJ, Slooter GD, Sonneveld DJA, Talsma AK, van Westreenen HL, Kusters M, Hompes R, Bonjer HJ, Sietses C, Tuynman JB (2020) Locoregional recurrences after transanal total mesorectal excision of rectal cancer during implementation. Br J Surg. https://doi.org/10.1002/bjs. 11525

31. Simo V, Tejedor P, Jimenez LM et al (2020) Oncological safety of transanal total mesorectal excision (TaTME) for rectal cancer: midterm results of a prospective multicentre study. Surg Endosc. https://doi.org/10.1007/s00464-020-07579-4

32. Aubert M, Mege D, Panis Y (2019) Total mesorectal excision for low and middle rectal cancer: laparoscopic versus transanal approach - a meta-analysis. Surg Endosc. https://doi.org/10.1007/ s00464-019-07160-8

33. Wasmuth HH, Færden AE, Myklebust $\mathrm{T}$ et al (2020) Transanal total mesorectal excision for rectal cancer has been suspended in Norway. Br J Surg 107:121-130

34. Berardi G, Aghayan D, Fretland A et al (2019) Multicentre analysis of the learning curve for laparoscopic liver resection of the posterosuperior segments. Br J Surg 106:1512-1522

35. Andreetti C, Menna C, Ibrahim M, Ciccone AM, D'Andrilli A, Venuta F, Rendina EA (2014) Postoperative pain control: videothoracoscopic versus conservative mini-thoracotomic approach. Eur J Cardio-thoracic Surg 46:907-912

36. Sood A, Meyer CP, Abdollah F, Sammon JD, Sun M, Lipsitz SR, Hollis M, Weissman JS, Menon M, Trinh QD (2017) Minimally invasive surgery and its impact on 30-day postoperative complications, unplanned readmissions and mortality. Br J Surg 104:13721381

37. Bona S, Molteni M, Rosati R, Elmore U, Bagnoli P, Monzani R, Caravaca M, Montorsi M (2014) Introducing an enhanced recovery after surgery program in colorectal surgery: a single center experience. World J Gastroenterol 20:17578-17587

38. Chemali ME, Eslik GD (2017) A meta-analysis: postoperative pain management in colorectal surgical patients and the effects on length of stay in an enhanced recovery after surgery (ERAS) setting. Clin J pain. https://doi.org/10.1097/AJP.0000000000000370

39. Aarts MA, Rotstein OD, Pearsall EA, Victor JC, Okrainec A, McKenzie M, McCluskey SA, Conn LG, McLeod RS (2018) Postoperative ERAS interventions have the greatest impact on optimal recovery. Ann Surg 267:992-997

Publisher's note Springer Nature remains neutral with regard to jurisdictional claims in published maps and institutional affiliations. 